\title{
MemLog, an Enhanced Lifelog Annotation and Search Tool
}

\author{
Lijuan Marissa Zhou, TengQi Ye, Brian Moynagh, Liting Zhou, and Cathal \\ Gurrin \\ Insight Centre for Data Analytics, \\ Dublin City University, Dublin 9, Ireland \\ mzhou@computing.dcu.ie
}

\begin{abstract}
As of very recently, we have observed a convergence of technologies that have led to the emergence of lifelogging as potentially pervasive technology with many real-world use cases. While it is becoming easier to gather massive lifelog data archives with wearable cameras and sensors, there are still challenges in developing effective retrieval systems. One such challenge is in gathering annotations to support user access or machine learning tasks in an effective and efficient manner. In this paper, we demonstrate a web based on-line annotation system for sensory and visual lifelog data and show it in operation on a large archive of nearly 1 million lifelog images and 27 semantic concepts in 4 categories.
\end{abstract}

\section{Introduction}

Digital recording of life experience is gaining popularity with the emergence of a new generation of wearable sensors. Lifelogging refers to the idea that an individual can utilise wearable sensors to generate a rich archive of life experience [2]. Typically this is done using a number of sensors, including a wearable camera. Developing effective lifelog search engines and management tools is very challenging for reasons such as the lack of available test collections and the difficulty of searching through multimodal data archives. Central to this challenge is the idea that sufficiently detailed annotations of lifelog data, both for use in supporting human analysis of lifelog data, or as a source of training data for machine-learning-based concept detection tools, are difficult, expensive and time-consuming to generate.

In this paper, we present a lifelog management framework called MemLog, which is based on extensive experience of managing and annotating lifelog archives. Using the MemLog system, lifelog data can be uploaded from a variety of lifelogging devices into a cloud-based service and annotated in an efficient manner, using both shared and personal concepts. Instead of simply displaying a sequential list of images for annotation, which can number many thousands per day, MemLog can compress consecutive images for efficient annotation and learn from user's individualized annotations to segment their lifelog recordings into multiple life events. Given a starting set of user annotations, MemLog suggests multiple most likely labels for each event based on an underlying machine 
learning technique. Although MemLog is designed for lifelog data management, it but also can be applied to other temporally organised multimedia content.

\section{Background}

Lifelogging is becoming a normative activity and much of the research into lifelogging has used such wearable cameras, along with other sensors to capture life experience in high-fidelity. Wearable cameras, such as the SenseCam are designed to automatically capture all aspects of wearer's day[3] in rich visual detail. When hung on a lanyard around the neck, the SenseCam could take photos automatically of the activities of the individual, from their viewpoint. Up to 4,000 pictures could be captured daily. There are many commercial offerings also, such as the Narrative Clip or OMG Autographer.

Hence, this has provided us with the ability to gather and store large volumes of personal data in a straightforward manner, using off-the-shelf tools or even smart-phones. Once the lifelog data is gathered, to be useful, it must be indexed in some manner and made available through an interface. There have been a number of lifelog data browsing interfaces developed. The first of these was the SenseCam image browser which facilitates annotations of images of interest and also rapid playback of image sequences from Hodges et al. [3]. Other systems include the event-based SenseCam image browser which automatically segments lifelog data into events or episodes and then allows users to manually annotate those events [1]. The authors have previously developed the ShareDay lifelog system that supports reminiscence through incorporating event segmentation and group sharing [5]. From all these tools, the only annotation tool for large lifelog archives is from Doherty et al. [1], which simply presents a sequence of images for multi-pass annotation. The contribution of the system proposed in this paper is that it is flexible to the individual annotation needs of the user, easy to maintain and manage and it actively utilises the user annotations in order to generate additional suggested concept annotations.

\section{MemLog Overview}

The MemLog system is an end-to-end lifelog management and annotation system and a screen-shot of MemLog is shown in Figure 1, in which an annotator can be seen annotating a piece of content. On the left of the screen is the calendar to access the lifelog data, on the right is the annotation panel and the main part of the screen is the data panel, which displays (in this case) a full-sized playback view of the event being annotated. MemLog has been designed with multiple attributes that meet practical research purposes, including both local and remote data uploading, manual and automatic data annotation, and quality checking etc. These attributes have been gathered after extensive consideration of the needs of large-scale lifelog annotation efforts. Aside from multiple user types, uploader tools for multiple lifelogging devices and annotation quality checks and 


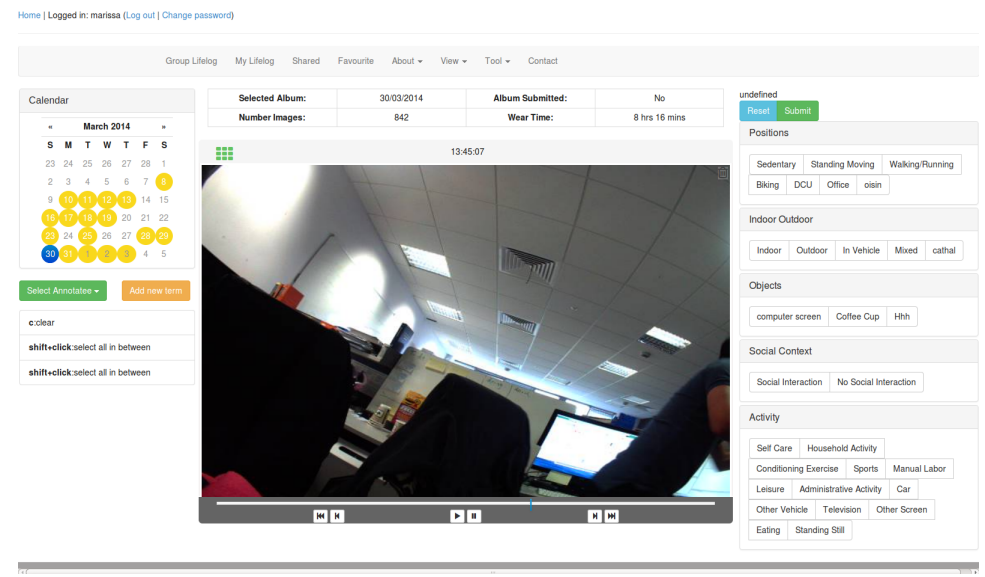

Fig. 1: MemLog in Use, the main interface with data picker and tag picker validations, the most relevant and important novel attributes of MemLog can be summarised thus:

- Extensible Categories and Concept Labels. Experiences of large-scale annotation suggests that a mix of shared and user-specific concepts is needed. Hence, a core set of 27 concepts is integrated in 5 categories. For personalised concept annotation, users can add their own personal label sets to the predefined concepts. Concepts can he hierarchical or independent.

- Automatic Label Recommendation. A lifelog for an individual user can surpass 4,000 images per day and poses major challenges for manual annotation [2], hence Memlog integrates the first lifelogging event-based automatic concept label suggestion tool, which is considered necessary for efficient and effective management of large volumes of content[4]. Events represent repeating life patterns and these events are detected automatically (using supervised machine learning) by MemLog and by computing the visual similarity of lifelog events, as well as the repeating patterns of user activity, labels for current lifelog events are recommended using previously annotated labels. Annotators can check and modify these recommended labels, reviewing them in a dedicated review screen for validation and update.

- Event Reviewing. Event reviewing shows storyboards of daily events to the user for review or annotation. All events are presented to users chronologically with key-frames of each event. Each event can be demonstrated either in a conventional thumbnail view or in movie view (sequential playback of lifelog images). Selecting an image allows the user overview all annotations assigned to that event, including more general details of events like start/end time and number of pictures in that event. Users can modify annotation if they think it is not proper for that event and add new concepts for that event if it is not automatically added. 


\subsection{Evaluation of Memlog}

We have tested Memlog on two data-sets, one is one month's data from 7 participants, another is one week's data of 46 users, and shown it to be effective. The annotation was performed by 6 interns who tested the system by uploading, annotating and conducting quality checking. Our findings show that dividing the annotation process into different categories can speed up by $70 \%$ of time and increase the effectiveness of the annotation process. In addition, the event segmentation technique with annotation label recommendation allows for significant increases in annotation speed as a benefit of tag recommendation.

\section{Conclusion}

There is a lack of a useful visual data annotation engine in the lifelog research area. In this paper, we introduced MemLog, a lifelog management system for lifelog researchers to annotate large volumes of lifelog data. We believe that the system is not only applicable for lifelog researchers, but also more generally for image crowdsourcing organizations, for reasons such as data annotation, training data gathering for machine learning and enhanced quality multimedia data retrieval. In the future, we are planning to continue using this system to facilitate additional multi-modal data research projects and are exploring how we can potentially make this software available for others to use. Any feedback in demonstration sessions will be very welcome.

\section{Acknowledgements}

This publication as emanated from research conducted with the financial support of Science Foundation Ireland, under grant nos. 11/RFP.1/CMS/3282 and 13/TIDA/I2875(FT).

\section{References}

1. A. R. Doherty, C. J. A. Moulin, and A. F. Smeaton. Automatically assisting human memory: A sensecam browser. Memory, 19(7):785-795, 2011.

2. C. Gurrin, A. F. Smeaton, and A. R. Doherty. Lifelogging: Personal big data. Foundations and Trends@ in Information Retrieval, 8(1):1-125, 2014.

3. S. Hodges, L. Williams, E. Berry, S. Izadi, J. Srinivasan, A. Butler, G. Smyth, N. Kapur, and K. Wood. Sensecam: A retrospective memory aid. In UbiComp 2006: Ubiquitous Computing, pages 177-193. Springer, 2006.

4. S. Sarin, M. Fahrmair, M. Wagner, and W. Kameyama. Holistic feature extraction for automatic image annotation. In Multimedia and Ubiquitous Engineering (MUE), 2011 5th FTRA International Conference on, pages 59-66. IEEE, 2011.

5. L. M. Zhou, N. Caprani, C. Gurrin, and N. E. O'Connor. Shareday: A novel lifelog management system for group sharing. In Advances in Multimedia Modeling, pages 490-492. Springer, 2013. 